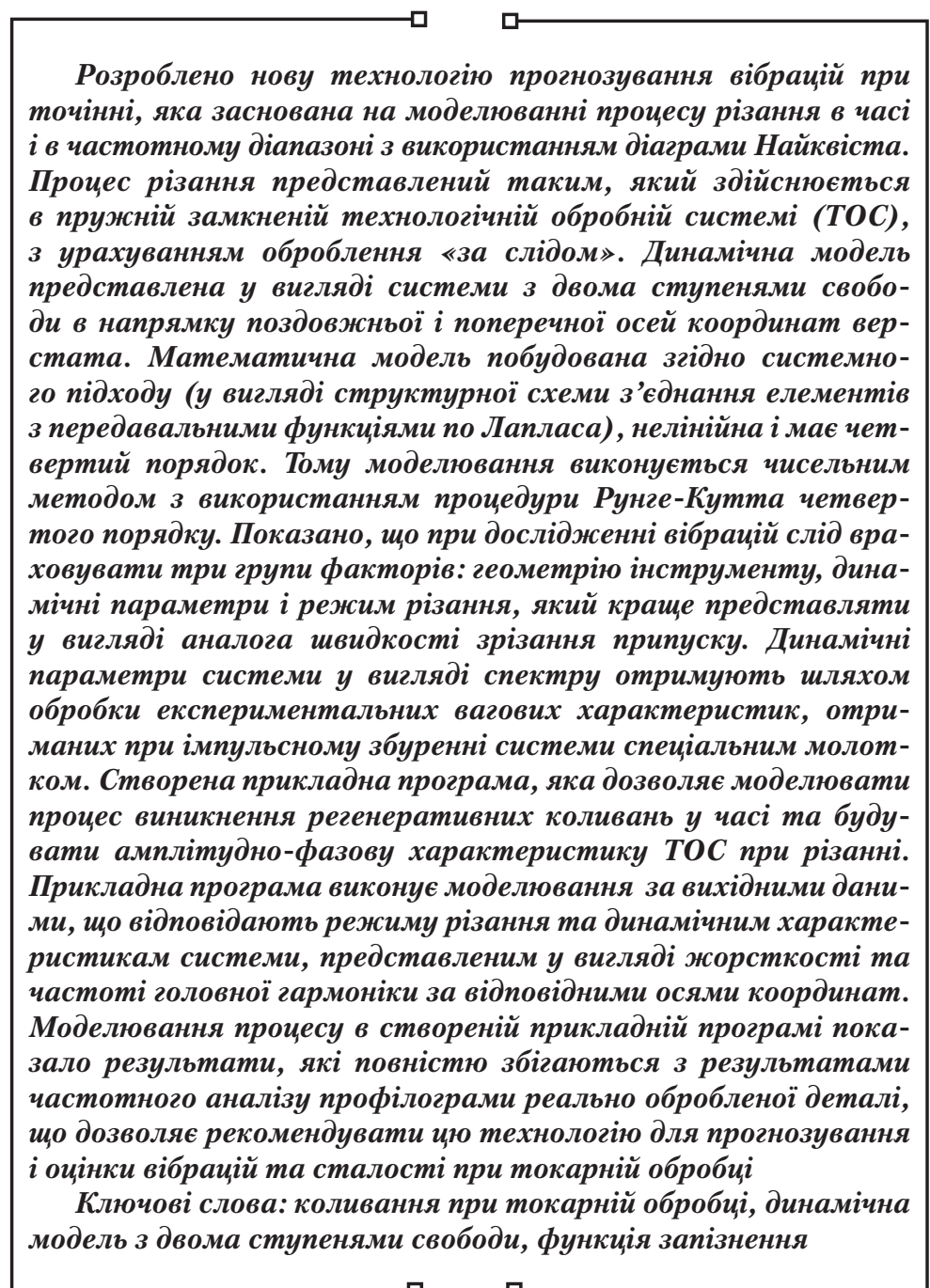
$\longrightarrow$

\section{PREDICTION OF CHATTER STABILITY IN TURNING}

\author{
Y. Petrakov
}

Doctor of Technical Sciences, Professor, Head of Department Department of Manufacturing

Engineering*

E-mail: ypetrakov.86@gmail.com

M. Da n y l c he n k o

Assistant

Department of Machine Tools and Machinery Systems* E-mail: rudenko_m@i.ua

A. P e t r y s h y n

$\mathrm{PhD}$, Senior Lecturer

Department of Applied

Hydro-Aeromechanics and Mechatronics*

E-mail: m.p3shka.a.i@gmail.com

*National Technical University of Ukraine «lgor Sikorsky Kyiv Polytechnic Institute» Peremohy ave., 37, Kyiv, Ukraine, 03056

Copyright (C) 2019, Y. Petrakov, M. Danylchenko, A. Petryshyn This is an open access article under the CC BY license (http://creativecommons.org/licenses/by/4.0)

\section{Introduction}

Cutting process is carried out in the elastic technological machining system (TMS) and it is closed-loop by its nature due to feedback between the defined parameter of cutting process (for example, depth of cut) and cutting force, which causes deformation variation, therefore, proportional change depth of cut variation, and consequently cutting force variation again, etc. In addition, usually cutting process is multiple-pass, with so-called machining «by trace». Many researchers consider that it is the main reason of vibrations and chatter on the part surface.

Regenerative oscillations (self-oscillations) have a significant influence on the process performance, lowers the machined part surface quality and dimensional accuracy. In addition, vibrations can lead to cutting tool deterioration or even machine tool itself, therefore, increasing manufacturing costs [1]. That is why studying and solving of the vibration problem during machining is very important for performance improvement of these operations. Usually, such study is performed using different modeling and analysis methods that focused on prediction of the best machining conditions.
Increasing the reliability and performance of such studies is still a timely scientific and technical issue.

\section{Literature review and problem statement}

Generally, most of researchers consider that vibrations in TMS occur due to the interaction of elastic system and cutting process. Thereby this interaction is the oscillator and the source of dynamic effects in the elastic system, which appears in periodic relative displacements of the tool and the workpiece. The main reason of vibrations occurrence in machining [2] is considered to be nonlinearity of cutting process characteristics, and the model is based on a one-degree-offreedom system, which decreases its adequacy.

Other researchers [3] take into account a complex of reasons, including a static and dynamic cutting force duality, the mutual influence of oscillations in the coordinate relationship, secondary excitation from machining by chatter. However, the model with one degree of freedom is taken, and the mathematical description is based on the dependence of the ultimate strength of metals on temperature. Such an 
approach cannot explain the oscillations in a system with a high frequency, since the inertia of such characteristic as temperature is known.

The fundamental research of vibrations in the system during cutting, presented in [4] should be noted. The proposed approaches are mainly based on the usage of analytical solution methods of differential equation system that does not exceed second order, while stability conditions determination is based on characteristic equation root analysis, which does not always provide adequate results [5]. In studies of the frequency domain of vibrations and cutting stability using the 'lobes-diagram' [6], despite a wide experiment, there is still no mathematical model to predict the occurrence of chatter.

When solving modeling problems, most of the studies suggest considering the time delay between sequential turns, which depends on spindle speed, which affects the phase between chatter waves on the machined surface [7]. The significance of this effect in the cutting process instability is also pointed out, but the presented mathematical models do not allow modeling the process in the time domain [8]. The problem of ensuring vibration-free machining can be solved using the system [9]. However, this method provides for complex research and requires significant costs. For example, in [10] it was noted that the experimental method is time consuming and expensive, while the analytical method eliminates the need for experiments. Nevertheless, the proposed analytical method cannot be considered exhaustive, since it is also based on an analysis of the roots of the characteristic equation.

Against this background, the need to develop models in the direction of increasing their adequacy is obvious, increasing the number of degrees of freedom of the dynamic system, which will make it possible to reliably and simply predict cutting conditions that ensure the stability of the process using simulation by numerical methods [11].

The analysis confirmed the main condition for adequate model creation. It is necessary to have:

- an adequate model of the dependence of the cutting force on the processing mode, workpiece material, tool and other factors;

- the dynamic characteristics of an elastic technological system;

- a geometric model of the interaction of the tool and the workpiece in the form of an analogue of the allowance cutting speed - Material Removal Rate [12].

\section{The aim and objectives of the study}

The aim of the study is to develop a mathematical model of the cutting process for turning taking into account machining by chatter trace and two degrees of freedom, to create information support for numerical simulation and system stability prediction.

This allows solving not only the problems of choosing the cutting mode, but also the design of the dynamic parameters of TMS to perform stable processing with maximum productivity.

To attain the aim, it is necessary to accomplish the following objectives:

- based on a systematic approach, to develop a structural diagram of the cutting process as a single-mass system with two inputs along orthogonal axes: depth of cut and length feed on revolve;
- to create an application program for numerical simulation in the time and frequency domains;

- to perform an experimental verification of theoretical results and to state practical recommendations for use in production.

\section{Methods and results of the turning process study}

\section{1. Problem statement}

Prediction of vibrational stability in machining turning involves the availability of a mathematical model, which consists of cutting force, elastic closed-loop dynamic system, and geometric interaction separate models. The mathematical model should combine these particular models into a single system with two inputs for cutting depth and length feed per revolution, as well as corresponding outputs which form the geometric parameters of the removed material layer. Modeling involves the use of numerical methods with some of the parameters of mathematical models set according to the results of experiments to ensure the adequacy.

Therefore, it is proposed to build an application program algorithm that is based on the developed mathematical model and implements modeling in the time and frequency domains. For the results analysis, it is proposed to develop criteria for the stability of the cutting process.

\section{2. Mathematical model of the turning process}

To determine the force characteristics of the turning process, the well-known empirical dependence of tangential cutting force on machining conditions can be used [13]:

$$
F_{z}=C_{P_{z}} h^{x_{P_{z}}} f^{y_{P_{2}}} V^{n_{P_{2}}} k
$$

where $C_{P z}$ - coefficient, which depends only on workpiece material; $k$ - correction index; $x_{P z}, y_{P z}, n_{P z}$ - exponents; $h, f$, $V$ - cutting mode - depth of cut $(\mathrm{mm})$, feed rate $(\mathrm{mm} / \mathrm{rev})$ and cutting speed $(\mathrm{m} / \mathrm{min})$.

Fig. 1 shows the diagram of elastic deformations in turning, where it is shown how elastic deformations $\delta h$ in the $Y$ axis direction (along the cutting depth) lead to variation of cutting width $\delta f$ and, as a result - removed material layer area. Elastic deformations in the $X$ axis direction do not cause a change in the depth of cut, but directly affect the area.

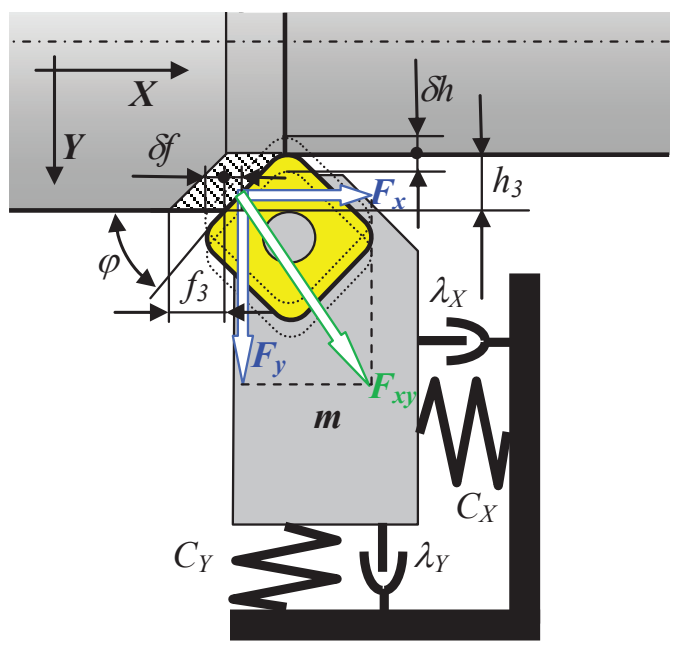

Fig. 1. Scheme of the elastic deformations influence on the removed material 
The cutting force components $F_{x}$ and $F_{y}$ are the decomposition vectors of the horizontal component $F_{x y}$, which acts along the front surface in the direction coinciding with the direction of the chip flow and the plan angle $\varphi$ of the cutting insert, and can be determined by $F_{x y}=0.6 F_{z}$ [13]. Thus, from the geometric relations in Fig. 1:

$$
F_{y}=0.6 F_{z} \cos \varphi, F_{x}=0.6 F_{z} \sin \varphi .
$$

In general, a mathematical model of the cutting process in elastic TMS can be represented in the form of a block diagram (Fig. 2).

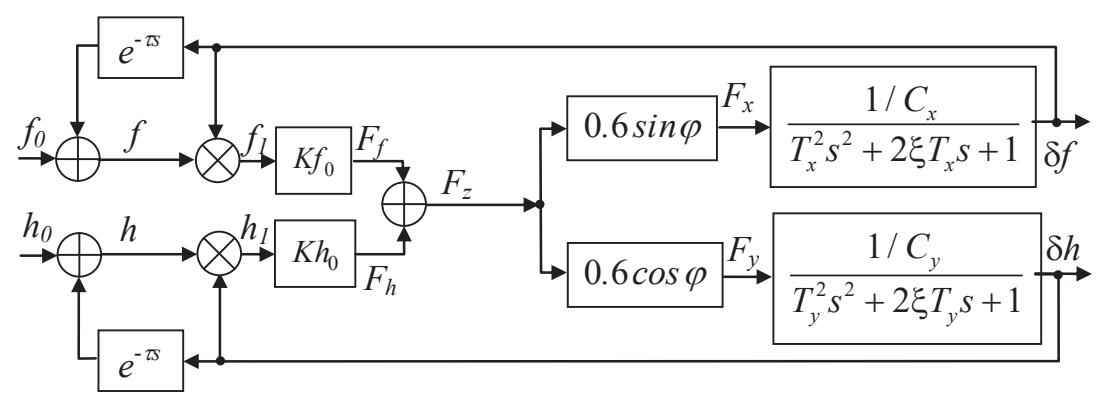

Fig. 2. Block diagram of the turning process

The block diagram represents a system with two inputs in depth $h_{0}$ and feed rate $f_{0}$ and, accordingly, two outputs - deformations $\delta h$ and $\delta f$ in these directions. The block diagram takes into account machining by chatter trace in the direction of two coordinates due to the delay transfer functions $e^{-\tau s}$, where $\tau$ - the time of one spindle revolution, $s-$ the Laplace operator. Therefore, the current feed is $f=f_{0}+\delta f e^{-\tau s}$, and the effective depth $h=h_{0}+\delta h e^{-\tau s}$, respectively, according to the notation on the block diagram.

Considering the relatively small values of deformations compared to the depth (width) of cut, the dependence of the cutting force (its $F_{Z}$ component) on the depth of cut $h$ and feed rate $f$ can be linearized:

$$
K h_{0}=\left(\partial F_{Z} / \partial h\right)_{0}=C_{p} x_{p} h_{0}^{x_{p}-1} f_{0}^{y_{p}} V_{0}^{n_{p}} k,
$$

and

$$
K f_{0}=\left(\partial F_{Z} / \partial f\right)_{0}=C_{p} h_{0}^{x_{p}} y_{p} f_{0}^{y_{p}-1} V_{0}^{n_{p}} k
$$

where $h_{0}, f_{0}, V_{0}$ - parameter values in the linearization point. Thus, the tangential component $F_{z}$ of the cutting force with this approach can be represented by the dependence from the block diagram: $F_{z}=f_{1} K f_{0}+h_{1} K h_{0}$.

The elastic dynamic system is represented by a singlemass model that is under the force excitation along two coordinates, which correspond with cutting force components. Since the parameters of the dynamic system must be determined experimentally, the transfer functions of the dynamic system can be conveniently represented as vibrational elements. The period $T$ of the elements oscillations is related to stiffness and reduced mass:

$$
T=2 \pi / \omega
$$

where $\omega$ - natural frequency ( $\mathrm{rad} / \mathrm{s})$.

Stiffness along the coordinate axes is determined experimentally, and the natural frequency is determined from the spectrum main harmonic of the system response to a pulse load, i. e. by its weight characteristic. The same characteristic is used for the oscillations damping coefficient $\xi$ determination in a similar way as for the vibrational element [11].

Modeling of such a system (Fig. 2) can be performed only by numerical methods in the time and frequency domains. According to the method, it is most convenient to present a mathematical model as a transfer function with Laplace images. To obtain the transfer function from input $f$ to output $\delta f$, it is necessary to use the connections of the block diagram in Fig. 2, setting $h=0$. In such case, the following system of equations can be obtained from the block diagram:

$$
\left\{\begin{array}{l}
f_{1}=f-\delta f, \\
F_{f}=f_{1} K f_{0}, \\
F_{h}=\delta h K h_{0}, \\
F_{z}=F_{f}-F_{h}, \\
\delta f=F_{z} 0.6 \sin \varphi \frac{1 / C_{x}}{T_{x}^{2} s^{2}+2 \xi T_{x} s+1}, \\
\delta h=F_{z} 0.6 \cos \varphi \frac{1 / C_{y}}{T_{y}^{2} s^{2}+2 \xi T_{y}+1} .
\end{array}\right.
$$

From the system of equations (6), by the substitution of variables method, we obtain an equation that relates the displacement $f$ in the $X$ axis direction (length feed per revolution) with the depth and deformation $\delta f$ in the same direction. From this equation, it is easy to obtain the desired transfer function:

$$
\begin{aligned}
& \frac{\delta f(s)}{f(s)}= \\
& =\frac{0.6 \sin \varphi K f_{0} W_{y} C_{y}}{W_{x} C_{x} W_{y} C_{y}-K f_{0} 0.6 \sin \varphi W_{y} C_{y}+W_{x} C_{x} 0.6 \cos \varphi K h_{0}},
\end{aligned}
$$

where

$$
W_{x}=T_{x}^{2} s^{2}+2 \xi T_{x} s+1, \quad W_{y}=T_{y}^{2} s^{2}+2 \xi T_{y} s+1 .
$$

After simple algebraic transformations, we have the transfer function in the standard form:

$$
W(s)=\frac{\delta f(s)}{f(s)}=\frac{\tau_{02}^{2} s^{2}+\tau_{12} s+k_{2}}{T_{02}^{4} s^{4}+T_{12}^{3} s^{3}+T_{22}^{2} s^{2}+T_{32} s+1},
$$

where time constants $\tau, T$ with corresponding indices and the coefficient $k_{2}$ are determined from equation (7), which is written in standard form in descending order of the Laplace operator $s$ powers.

For system modeling in the time domain by the numerical method using the standard 4th-order Runge-Kutta numerical integration procedure, equation (8) of motion along the input $f$ must be represented in the state variables in the matrix form:

$$
\left\{\begin{array}{l}
s U[1]=-A_{1} U[1]+U[2], \\
s U[2]=-A_{2} U[1]+A_{5} f+U[3], \\
s U[3]=-A_{3} U[1]+A_{6} f+U[4], \\
s U[4]=-A_{4} U[1]+A_{7} f,
\end{array}\right.
$$


where

$$
\begin{aligned}
& A_{1}=T_{12}^{3} / T_{02}^{4}, A_{2}=T_{22}^{2} / T_{02}^{4}, \\
& A_{3}=T_{32} / T_{02}^{4}, A_{4}=1 / T_{02}^{4}, \\
& A_{5}=\tau_{02}^{2} / T_{02}^{4}, A_{6}=\tau_{12} / T_{02}^{4}, \\
& A_{7}=k_{2} / T_{02}^{4},
\end{aligned}
$$

$s U, U-$ state variable arrays.

\section{Experimental studies of TMS dynamic characteristics}

The main goal of experimental research is to confirm the adequacy of the developed mathematical model. Since the mathematical model includes such parameters as the stiffness of the TMS, natural frequency and the vibration damping coefficient, it was decided to carry out full-scale experiments for adjustment, performing measurements in the places and directions indicated by the arrows, the scheme of adjustment is shown in Fig. 3.

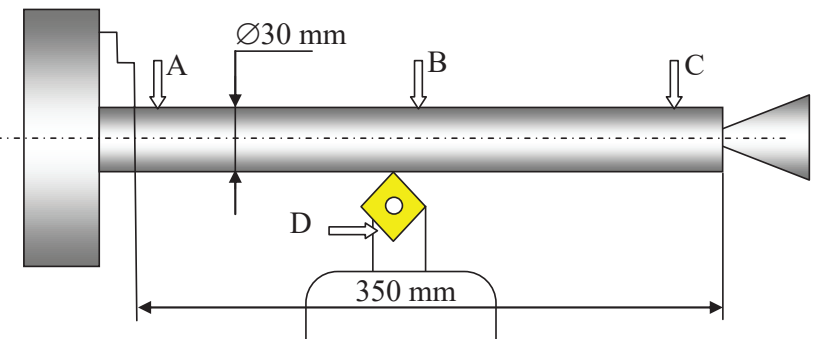

Fig. 3. Measurement scheme

The stiffness was measured under load in the indicated directions through the tensometric dynamometer UDM600 (All-Russian Research Institute, Russian Federation) by force up to $1,200 \mathrm{~N}$, while displacement was determined by measuring head with a division value of $0.01 \mathrm{~mm}$. System dynamic characteristics at these points were determined by digital arrays of weight characteristics, with signal decomposition (Fig. 4, $a$ ) in the frequency domain and obtaining its spectrum (Fig. 4, $b$ ).

The weight characteristic is the response of the system to the impulse load as a result of an impact by a special hammer in the places highlighted in Fig. 3.
The standard equipment was used for vibration measurements with a piezoelectric accelerometer PCB 353B15, an amplifier PBC 480E09 with an NI USB-9215 ADC (Piezotronics, Inc., USA). Digital signal processing was performed using the FFT algorithm (Fast Fourier Transform) in MATLAB. The measurement results are presented in Table 1, indicating the peak harmonics for the obtained spectra, and the frequencies with the maximum signal amplitude are shown in bold.

Table 1

Experimental data

\begin{tabular}{|c|c|c|c|c|c|}
\hline \multirow{2}{*}{ Point } & \multirow{2}{*}{$\begin{array}{c}\text { Stiffness, } \\
\text { N/mm }\end{array}$} & & \multicolumn{4}{|c|}{ Frequency, Hz } \\
\cline { 3 - 6 } & & I & II & III & IV \\
\hline$A$ & 11.617 & $\mathbf{4 7 3}$ & 832 & 2.421 & - \\
\hline$B$ & 6.936 & 250 & 474 & $\mathbf{1 . 6 4 9}$ & 2.277 \\
\hline$C$ & 9.468 & 235 & 472 & $\mathbf{5 9 3}$ & 1.757 \\
\hline$D$ & 3.723 & 1.100 & $\mathbf{2 . 1 5 4}$ & - & - \\
\hline
\end{tabular}

Machining of the steel 20 workpiece was carried out with a passing cutter with a cutting edge angle of $45^{\circ}$, cutting mode: depth of cut $2.5 \mathrm{~mm}$, feed rate $0.2 \mathrm{~mm} / \mathrm{rev}$, spindle speed 1,600 rpm. The cutting process was accompanied by sound vibrations at a high frequency and tangible vibrations, traces of which are visible on the machined surface.

In the selected areas of the machined part $(A, B, C$ in Fig. 3), cross-section roughness measurements were carried out on the Talyrond roundness gage and their frequency analysis was performed.

Fig. 5, $a$ shows the graph of the digital signal of the surface profile in section $C$, and Fig. $5, b$ shows the spectrum of signal decomposition in the frequency domain, considering that the profile was formed during one revolution equal to $0.0375 \mathrm{~s}$.

It is clear that the surface geometry (macro- and microroughness) is formed as a result of the relative movement of the tool and the part, which occurs due to TMS fluctuations during cutting.

Therefore, the spectral decomposition of the profile, taking into account the spindle speed, can serve as a reliable criterion for the adequacy of the created mathematical model of the process. In other words, the equivalence between the spectrum and the amplitude-phase characteristic of the model should be satisfied.

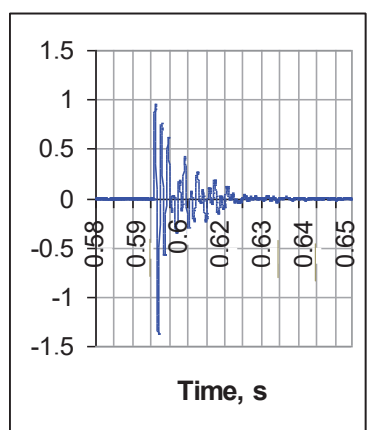

$a$

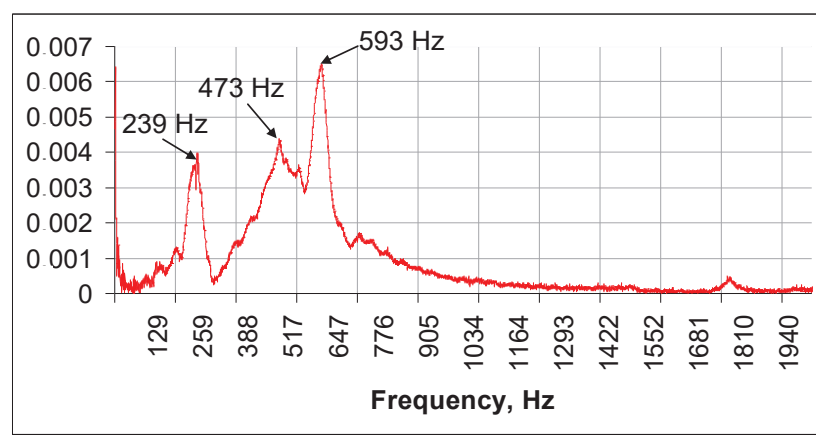

$b$

Fig. 4. System characteristics in point $C: a-$ weight characteristic; $b-$ spectrum 


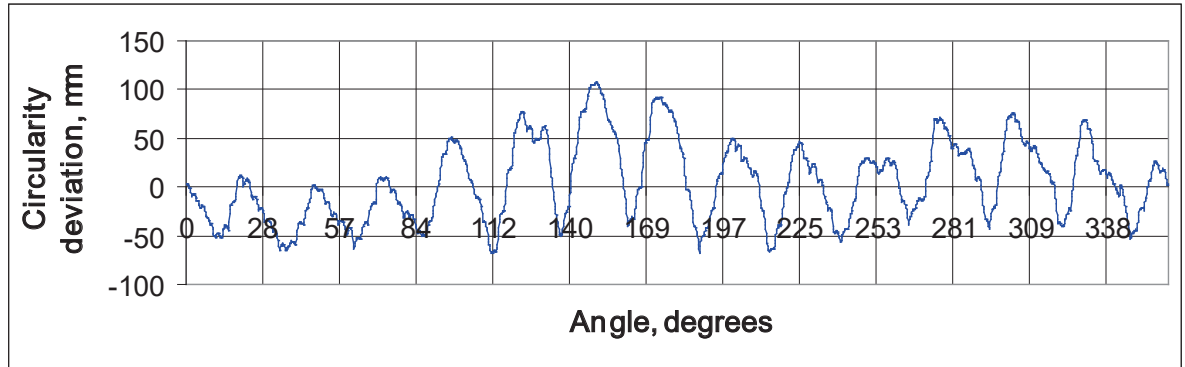

$a$

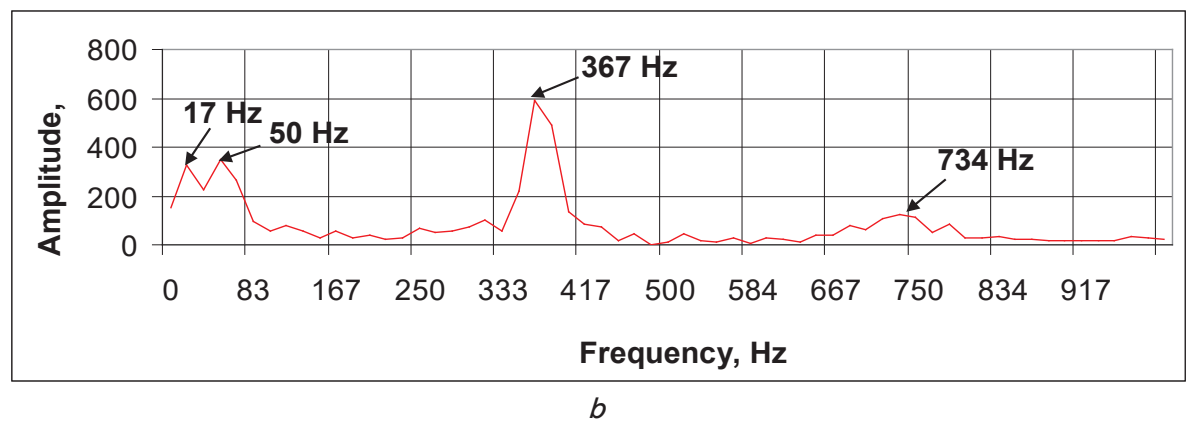

Fig. 5. Results of workpiece profile measurement in point $C$ : $a-$ Talyrond trace, $b-$ spectrum

\section{Modeling of the transfer and frequency characteristics of the process}

For the cutting process simulation in turning, an application program, the core of which is the mathematical model developed above has been created. The application program allows simulation of the process, both in the time and in the frequency domains as an amplitude-phase characteristic. Fig. 6 shows the program interface for the cutting process modeling with dynamic parameters obtained experimentally for section $C$ (Fig. 3). The simulation is performed numerically using the fourth-order Runge-Kutta integration procedure, with an integration step of $0.00001 \mathrm{~s}$.

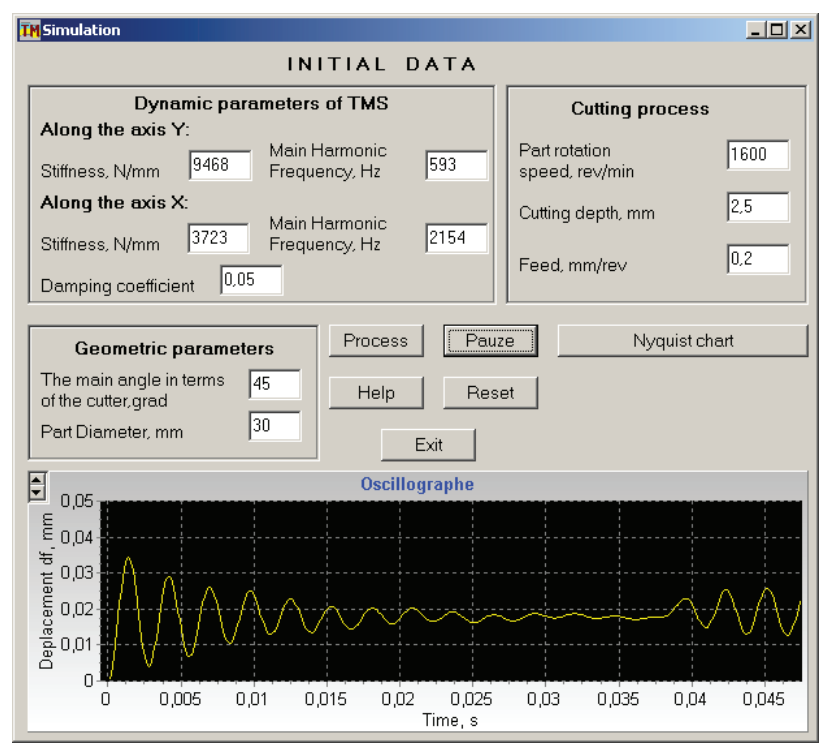

Fig. 6. Simulation program interface

The process begins with fluctuations caused by a spasmodic change in feed per revolution, which gradually fade. It corresponds to typical dynamic influence by a signal in the form of a Heaviside function in order to obtain a transient response. After the completion of one turn of the workpiece (at 1,600 rpm, it is $0.0375 \mathrm{~s}$ ), the system oscillations appear again, which is caused by regenerative excitation due to machining «by trace». However, in simulation these conditions with a time flow lead to the process attenuation.

With an increase in the cutting process intensity, for example, by increasing the depth of cut to $3.6 \mathrm{~mm}$, the simulation shows an increase in the oscillation amplitude, which indicates a possible loss of stability. Thus, the most important indicator of TMS can be determined as the maximum depth of cut, which determines the maximum possible metal removal performance.

The adequacy of the created software was verified by comparison of the developed TMS model frequency characteristics with the frequency analysis results of the surface profilogram of the actually machined part surface. By clicking on the «Nyquist Diagram» button, an amplitude-phase characteristic graph on the complex plane appears in a separate window (Fig. 7).

On the amplitude-phase characteristic curve, design points with the corresponding frequency in $\mathrm{Hz}$ are highlighted. The graph of the characteristic is plotted automatically as a spline, with the control points calculated numerically [11]. The maximum TMS oscillation amplitude can be observed at a frequency of $\sim 360 \mathrm{~Hz}$ (Fig. 7), which correlates well with the signal spectrum of the machined surface profile - main harmonic is located at this frequency (Fig. 5, b). Thus, the coincidence of the frequencies of the main, dominant harmonic of the model and the profile spectrum indirectly confirms the adequacy of the created mathematical model.

Simulation of the cutting process in the created program with parameters corresponding to the point $A$ demonstrated the frequency response shown in Fig. 8. It can be seen that the maximum amplitude of oscillations occurs at a frequency of 2,260 $\mathrm{Hz}$ (Fig. 8, $a$ ), which corresponds to the surface geometry left on the part when removing the cutter (Fig. 8, b). Traces of TMS oscillations in the amount of 82 waves are visible on the surface, which at a speed of 1,600 rpm corresponds to a frequency of 2,200 Hz, which also confirms the adequacy of the developed mathematical model and the created application program. 


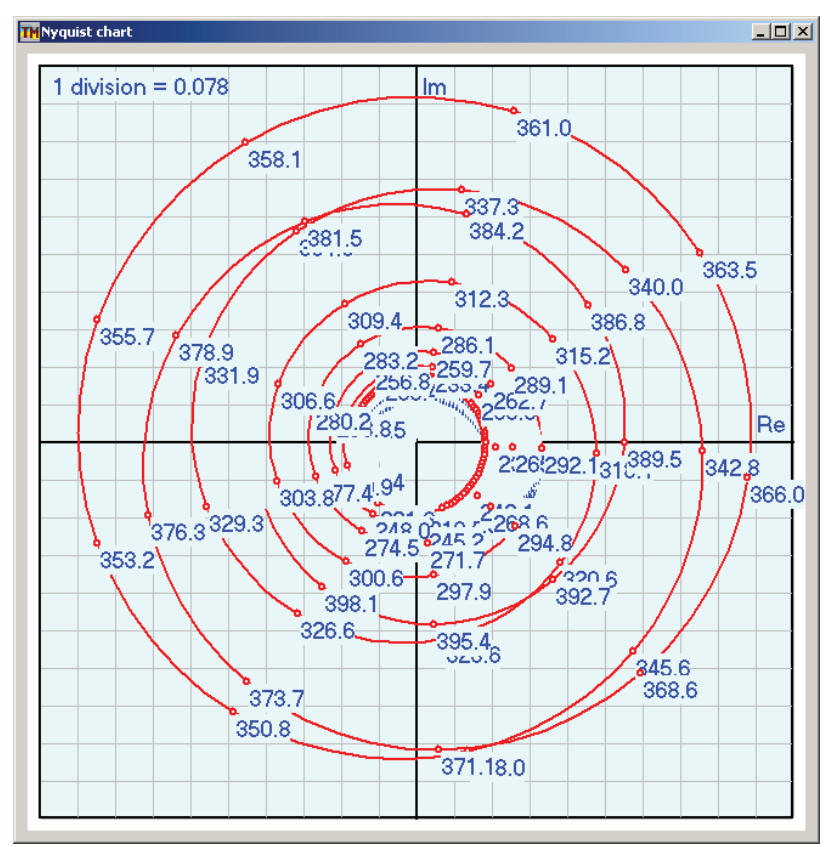

Fig. 7. Amplitude-phase characteristic of the TMS in turning (in point $C$ )

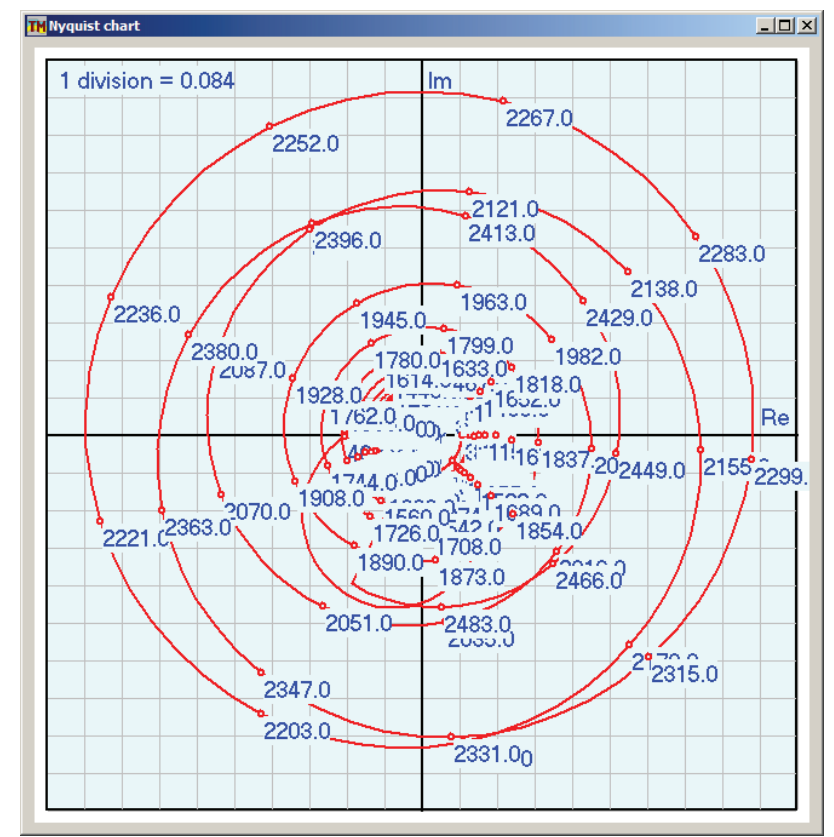

$a$

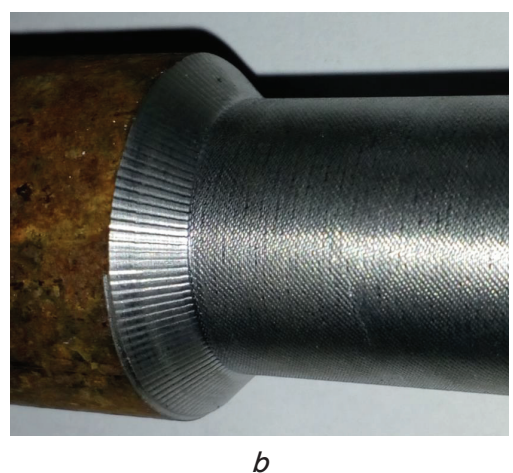

Fig. 8. Modeling and experimental results: $a-$ amplitude-phase characteristic of TMS in machining (point $A$ ), $b$-chatter on the part surface

\section{Discussion of vibration stability prediction results in turning}

The cutting process, in particular, during turning, is performed in an elastic closed-loop TMS and is always accompanied by vibrations. The amplitude of these vibrations forms the micro-roughness of the profile, and under certain conditions it can lead to stability loss of the cutting process. In any case, it is the amplitude of TMS vibrations that determines its maximum possible efficiency by the maximum cutting depth. Therefore, it is important to have tools and technologies for predicting the vibration resistance of the cutting process.

A new technology of prediction is proposed that is based on the mathematical model of elastic TMS with two degrees of freedom (Fig. 1, 2), which is described by a fourth-order transfer function with a delayed argument. Prediction is performed in the application program, considering three groups of factors, which are determined by experimental data: tool geometry, dynamic parameters of TMS (Table 1) and cutting mode. The model was verified for adequacy by comparing the spectrum profile of the micro-roughness of the actually machined surface (Fig. 5) with the amplitude-phase characteristic of the developed model (Fig. 7). The created application program allows evaluating the stability of TMS during cutting, as well as building a stability chart (the so-called «lobes-diagram») in the coordinates «depth of cut - cutting speed». Evaluation can be performed by observing the process in time, or in the frequency domains using the Nyquist criterion, taking into account the presence of a time lag element in the system [12]. An increase in the adequacy of the proposed model should be sought in increasing the number of masses and degrees of mobility of the dynamic system, as evidenced by the spectrum profile of the machined surface of the part (Fig. 5, $b$ ), where at least four significant harmonics can be distinguished.

For the development of this study, we can suggest the use of the created application program directly in the CNC machine rack to predict the likelihood of vibrations, the amplitude of which exceeds a predetermined level, when performing any control program for processing the part.

\section{Conclusions}

1. A mathematical model of the cutting process in an elastic closed-loop technological system taking into account machining by chatter trace has been developed. The model represents a dynamic system with two degrees of freedom and is formed as a fourth-order transfer function, which allows the use of numerical methods for system characteristics obtaining, both in time and in the frequency domains.

2. A new technology of vibration stability prediction in turning is proposed. The technology consists in the application of the created applied modeling program, which uses the initial settings (initial data) obtained as a result of experimental studies of the TMS dynamic parameters. Modeling in the application program allows building a stability chart in the coordinates «depth of cut - cutting speed».

3. The experimental studies of comparing the microroughness spectrum of the machined surface and the amplitude-phase characteristic of TMS confirmed the adequacy of the mathematical model in terms of coincidence of the main harmonic of the micro-roughness spectrum and the model amplitude-frequency characteristic that allow recommending the developed technology for practical use. 


\section{References}

1. Nicolescu, M., Frangoudis, C., Semere, D., Archenti, A., Rashid, A. (2015). New paradigm in control of machining system's dynamics. Journal of Machine Engineering, 15 (3), 117-137.

2. Moon, F. C., Kalmár-Nagy, T. (2001). Nonlinear models for complex dynamics in cutting materials. Philosophical Transactions of the Royal Society of London. Series A: Mathematical, Physical and Engineering Sciences, 359 (1781), 695-711. doi: https:// doi.org/10.1098/rsta.2000.0751

3. Korendyasev, G. K. (2013). On the physical models of auto-oscillations at the processing of metal. Vestnik nauchno-tehnicheskogo razvitiya, 7 (71), 15-25.

4. Altintas, Y. (2012). Manufacturing automation: metal cutting mechanics, machine tool vibrations, and CNC design. Cambridge University Press, 366. doi: https://doi.org/10.1017/cbo9780511843723

5. Cypkin, J. S. (1946). Stability of systems with retarding ferd-back. Avtomat. i Telemekh., 7 (2-3), 107-129. Available at: http:// www.mathnet.ru/links/5ee3c3be11a608350b9a033b7920668b/at13125.pdf

6. Eynian, M. (2014). Frequency Domain Study of Vibrations above and under Stability Lobes in Machining Systems. Procedia CIRP, 14, 164-169. doi: https://doi.org/10.1016/j.procir.2014.03.068

7. Mann, B. P., Patel, B. R. (2010). Stability of Delay Equations Written as State Space Models. Journal of Vibration and Control, 16 (7-8), 1067-1085. doi: https://doi.org/10.1177/1077546309341111

8. Stephenson, D. A., Agapiou, J. S. (2016). Metal Cutting Theory and Practice. CRC Press, 969. doi: https://doi.org/ 10.1201/9781315373119

9. Virtual CNC. Available at: https://www.malinc.com/products/virtual-cnc/

10. Palpandian, P., Prabhu Raja, V., Satish Babu, S. (2013). Stability Lobe Diagram for High Speed Machining Processes: Comparison of Experimental and Analytical Methods - A Review. International Journal of Innovative Research in Science, Engineering and Technology, 2 (3), 747-752.

11. Petrakov, Yu. V., Drachev, O. I. (2014). Avtomaticheskoe upravlenie protsessami rezaniya. Stariy Oskol: TNT, 408.

12. Tang, A., Liu, Z. (2008). Three-dimensional stability lobe and maximum material removal rate in end milling of thin-walled plate. The International Journal of Advanced Manufacturing Technology, 43 (1-2), 33-39. doi: https://doi.org/10.1007/ s00170-008-1695-y

13. Mazur, N. P., Vnukov, Yu. N., Grabchenko, A. I., Dobroskok, V. L., Zaloga, V. A., Novoselov, Yu. K., Yakubov, F. Ya.; Mazur, N. P., Grabchenko, A. I. (Eds.) (2013). Osnovy teorii rezaniya materialov. Kharkiv: NTU «KhPI», 534.

14. Trybrat, K. O., Petrakov, Yu. V. (2019). Modeliuvannia avtokolyvan pry tokarnomu obroblenni. Naukovi konferentsiyi Ukrainy, innovatsiyi molodi v mashynobuduvanni 2019. Available at: http://imm-mmi.kpi.ua/imm2019/paper/view/17039 